\title{
Role of Magnetic Resonance Imaging in Differentiating Spondylitis from Vertebral Metastasis
}

\author{
Yuyun Yueniwati, Dhanti Erma Widhiasi \\ Radiology Department, Medical Faculty, Universitas of Brawijaya, Malang, Indonesia
}

Study Design: Observational analytic design with a cross-sectional approach.

Purpose: To analyze the suitability of magnetic resonance imaging (MRI) in distinguishing radiology images with a corresponding delineation of spondylitis and vertebral metastasis confirmed by histology results.

Overview of Literature: MRI is an accurate modality for assessing vertebrae and their disorders. Infections and metastasis are most commonly found in the vertebrae. It is difficult to differentiate between these two disorders both clinically and radiographically, particularly in atypical cases.

Methods: McNemar statistical test was used to analyze the data. Samples were chosen using the consecutive method. There were 35 samples (14 males and 21 females), consisting of 22 samples of spondylitis and 13 samples of metastasis confirmed on histology examination.

Results: Nineteen (86\%) out of the 22 samples of histological spondylitis were diagnosed as having spondylitis on MRI, whereas all 13 samples of metastasis were $100 \%$ accurately diagnosed on MRI.

Conclusions: There was no statistically significant difference between diagnostic radiology using MRI and histological diagnosis with a $p=0.250$ ( $p>0.05$ ). In this respect, MRI was more precise in diagnosing metastasis. Typical MRI description of spondylitis was the involvement of anterior vertebrae and components of intervertebral discs, stiffening of discs, paravertebral abscess, and involvement of the vertebral segment sequence. Typical MRI delineation of metastasis was involvement of the anterior posterior vertebral component, paravertebral mass, and skip lesions.

Keywords: Histology; Magnetic resonance imaging; Metastasis; Spondylitis

\section{Introduction}

Magnetic resonance imaging (MRI) is an accurate imaging modality for assessing vertebral abnormalities since it has high sensitivity in detecting small changes in the fat and water components of the medullary bone, intervertebral discs, spinal cord, and soft tissues around the vertebrae [1,2]. Therefore, MRI is a powerful diagnostic tool for the evaluation of various vertebral abnormalities and for the differentiation of pathological features, which appear in each distinct process [3-7].

Vertebrae are the most common sites of infection, which account for about $2 \%$ to $7 \%$ of all cases of musculoskeletal infections. The incidence increases along with the

Received Jan 20, 2015; Revised Feb 25, 2015; Accepted Feb 27, 2015

Corresponding author: Yuyun Yueniwati

Radiology Department, Medical Faculty, Universitas of Brawijaya, Jl Veteran, Malang 65145, Indonesia

Tel:+62-341-569117, Fax: +62-341-341808, E-mail: yyueniwati@yahoo.com 
increase in age, i.e., from $0.3 \%$ per 100,000 population at the age of less than 20 years to $6.5 \%$ per 100,000 population at the age of 70 years [8-10].

Besides, vertebrae are the most common sites of metastasis to bone. Epidemiological data indicate that the incidence of metastasis to the vertebral column ranges from $30 \%$ to $70 \%$ in patients with primary malignancies. The prevalence of vertebral metastasis is 0.74 per 100,000 population. Further, data reveal that metastasis is a tumor, which develops in most vertebral columns as confirmed by new cases which are more frequently diagnosed every year $[4,11,12]$.

It is important to differentiate spondylitis from vertebral metastasis as there are significant differences in the subsequent management. Identification of both diseases by an accurate early diagnosis can reduce the disability and malfunctioning, as well as improve the quality of life and life expectancy of patients. In addition, establishment of an accurate early diagnosis can reduce the wide range of investigations performed for the diagnosis with the result that hospitalization and treatment costs can be reduced [3,11,13-15].

Nevertheless, it is difficult to differentiate these two disorders, both clinically and radiographically. Difficulties arise especially when the classic picture is not observed or when unusual patterns emerge (atypical picture). Atypical MRI images, either due to infections or vertebral metastasis, should be identified properly to avoid missing the diagnosis $[6,10]$.

This study was conducted to assess the radiological differences on MRI between spondylitis and vertebral metastasis confirmed by histology results in Dr. Saiful Anwar General Hospital (Malang, Indonesia). There is a greater tendency among clinicians to rely on MRI imaging modality for determining the diagnosis of spondylitis and vertebral metastasis with the expectation to improve the accuracy of diagnosis so that the subsequent management can be determined precisely.

\section{Materials and Methods}

Research design used was the observational analytic design with a cross sectional study approach. Data were collected retrospectively from medical records and MRI images of vertebrae and histology preparations in the department of radiology and pathology anatomy Saiful Anwar General Hospital from January 2010 to December
2013.

Samples were obtained using the consecutive sampling technique, with the following inclusion criteria: results of MRI examination of vertebrae on T1-weighted images (T1WI), T2-weighted images (T2WI), and T1WI with contrast administration with a diagnosis of spondylitis and vertebral metastasis, and age greater than 20 years. The exclusion criteria were: patients with previous vertebral abnormalities (congenital anomalies, primary vertebral tumor, and severe scoliosis), metabolic bone disorders, simultaneously occurring spondylitis and metastasis.

MRI images were interpreted by three experienced radiologists who were unaware of the results of histological examination in the patients. After collection of the data, the next process was tabulation of different test results (results of MRI and histology diagnostic test), which were presented in a $2 \times 2$ table.

\section{Results}

Based on the retrospective data, 35 samples which met the inclusion criteria were selected. The samples consist from both sexes, male and female, with an age range from 20 to 72 years.

Spondylitis was observed in samples from younger patients, i.e., patients with an average age of $37.7 \pm 13.4$ years. Metastasis was more prevalent in samples from older patients, i.e., patients with an average age of $51.5 \pm 12.8$ years. Both spondylitis and metastasis were more commonly found in samples from females than in samples from males at a prevalence rate of $63.6 \%$ and $53.8 \%$, respectively. The most common site for both spondylitis and metastasis was thoracic vertebrae. The prevalence of spondylitis and metastasis was $54.5 \%$ and $53.8 \%$, respectively. The highest signal intensity of spondylitis and metastasis on T1WI was hypointense, with a prevalence of $86.4 \%$ and $76.9 \%$, respectively. The highest signal intensity of spondylitis on T2WI was hyperintense, with a prevalence of $90.9 \%$. With respect to metastasis, the prevalence of hyperintense and isointense signal intensity was the same, i.e. $38.5 \%$ (Table 1).

The results of T1WI with contrast sequence indicated that spondylitis showed rims enhancement in $81.8 \%$ samples, while metastasis showed heterogeneous enhancement in $80.0 \%$ samples (Table 2).

The anterior vertebral component was more frequently 
Table 1. Sample distribution based on magnetic resonance imaging signal intensity characteristics

\begin{tabular}{lcc}
$\begin{array}{l}\text { Abnormality } \\
\text { T1-weighted images }\end{array}$ & $\begin{array}{c}\text { Spondylitis } \\
(\mathrm{n}=22)\end{array}$ & $\begin{array}{c}\text { Metastasis } \\
(\mathrm{n}=13)\end{array}$ \\
\hline Hypointense & $19(86.4)$ & $10(76.9)$ \\
\hline Hyperintense & $1(4.5)$ & $0(0)$ \\
\hline Isointense & $2(9.1)$ & $3(23.1)$ \\
\hline T2-weighted images & & $3(23.1)$ \\
\hline Hypointense & $1(4.5)$ & $5(38.5)$ \\
\hline Hyperintense & $20(90.9)$ & $5(38.5)$ \\
\hline Isointense & $1(4.5)$ &
\end{tabular}

Values are presented as number (\%).

Table 2. Sample distribution based on characteristics of contrast enhanced magnetic resonance imaging

\begin{tabular}{lccc} 
Abnormality Homogeneous & Heterogeneous & $\begin{array}{c}\text { Rim } \\
\text { enhancement }\end{array}$ \\
$\begin{array}{l}\text { Spondylitis } \\
\text { (n=22) }\end{array}$ & $1(4.6)$ & $3(13.6)$ & $18(81.8)$ \\
$\begin{array}{l}\text { Metastasis } \\
(n=13)\end{array}$ & $1(7.7)$ & $12(92.3)$ & 0 \\
\hline
\end{tabular}

Values are presented as number (\%).

involved in spondylitis, and the prevalence was $81.8 \%$. On the other hand, the anteroposterior component was more frequently involved in metastasis with a prevalence of 92.3\% (Table 3).

The prevalence of the largest anterior compression in spondylitis was $77.3 \%$, while the prevalence of the most central compression in metastasis was $76.92 \%$ (Table 4).

The results of T1WI with contrast sequence demonstrated that the commonest abnormality of spondylitis was rim enhancement with a prevalence of $81.8 \%$. Concurrently, the most frequently found intensification patterns in metastasis were heterogeneous enhancement, with a prevalence of $80.0 \%$ (Table 5).

Table presenting the distribution of MRI abnormalities shows that most images of spondylitis demonstrated end plate irregularities at a prevalence rate of $86.4 \%$, followed by intervertebral disc narrowing at a prevalence rate of $81.8 \%$, paravertebral mass at a prevalence rate of $95.5 \%$, paravertebral abscess at a prevalence rate of $86.4 \%$, and involvement of the sequential vertebral segments at a prevalence rate of $86.4 \%$. Further, MRI abnormalities
Table 3. Sample distribution based on characteristics of involved vertebral components

\begin{tabular}{lcr} 
Abnormality & Anterior & Anteroposterior \\
\hline Spondilitis $(\mathrm{n}=22)$ & $18(81.8)$ & $4(18.2)$ \\
\hline Metastasis $(\mathrm{n}=13)$ & $1(7.7)$ & $12(92.3)$ \\
\hline
\end{tabular}

Values are presented as number (\%).

Table 4. Sample distribution based on characteristics of vertebrae body compression

\begin{tabular}{lcc} 
Characteristic & $\begin{array}{c}\text { Spondylitis } \\
(\mathrm{n}=22)\end{array}$ & $\begin{array}{c}\text { Metastasis } \\
(\mathrm{n}=13)\end{array}$ \\
\hline Anterior & $17(77.3)$ & 0 \\
Central & $4(18.2)$ & $10(76.92)$ \\
Posterior & $1(4.5)$ & 0 \\
Anteroposterior & 0 & $3(23.08)$
\end{tabular}

Values are presented as number (\%).

Table 5. Sample distribution based on characteristics of intervertebral discs intensity

\begin{tabular}{lccc} 
Abnormality & Normal & Decreasing & $\begin{array}{c}\text { Contrast } \\
\text { intensifying }\end{array}$ \\
Spondylitis $(\mathrm{n}=22)$ & $2(9.1)$ & $2(9.1)$ & $18(81.8)$ \\
Metastasis $(\mathrm{n}=13)$ & $11(84.6)$ & $2(15.4)$ & 0 \\
\hline
\end{tabular}

Values are presented as number (\%).

in images of metastasis showed paravertebral mass at a prevalence rate of $92.3 \%$, and skip lesions at a prevalence rate of $61.5 \%$ (Table 6, Figs. 1, 2).

\section{Result analysis}

Result analysis by McNemar test showed that there was no significant difference between MRI and histology diagnosis with a $p=0.250(p>0.05)$. Diagnosis based on MRI for examining spondylitis showed that three errors had occurred (13\%). At the same time, in the case of metastasis, all samples (100\%) could be accurately assessed radiologically.

\section{Discussion}

The total number of samples included in this research was 35 , consisting of 22 samples of spondylitis and 13 samples of metastasis. The results showed that based on the sample 
Table 6. Sample distribution based on magnetic resonance imaging abnormalities

\begin{tabular}{lcccc} 
& \multicolumn{1}{c}{ Spondylitis (n=22) } & \multicolumn{1}{c}{ Metastasis (n=13) } \\
\cline { 2 - 4 } Abnormality & Available & Not available & Available & Not available \\
\hline End plate irregularity & $19(86.4)$ & $3(13.6)$ & 0 & $13(100.0)$ \\
Narrowing of discs & $18(81.8)$ & $4(18.2)$ & 0 & $13(100.0)$ \\
\hline Paravertebral mass & $20(90.9)$ & $2(9.09)$ & $12(92.3)$ & $1(7.7)$ \\
\hline Paravertebral abscess & $19(86.4)$ & $3(13.6)$ & $13(100.0)$ & $13(100.0)$ \\
Spinal canal stenosis & $22(100.0)$ & 0 & $13(100.0)$ & 0 \\
Abnormalities of spinal medulla signal & $22(100.0)$ & 0 & $8(61.5)$ & $5(38.5)$ \\
\hline Skip Lesion & $3(13.6)$ & $19(86.4)$ & $3(13.6)$ & $8(61.5)$ \\
\hline Involvement of sequential vertebral segment & $19(86.4)$ & & 0
\end{tabular}

Values are presented as number (\%).
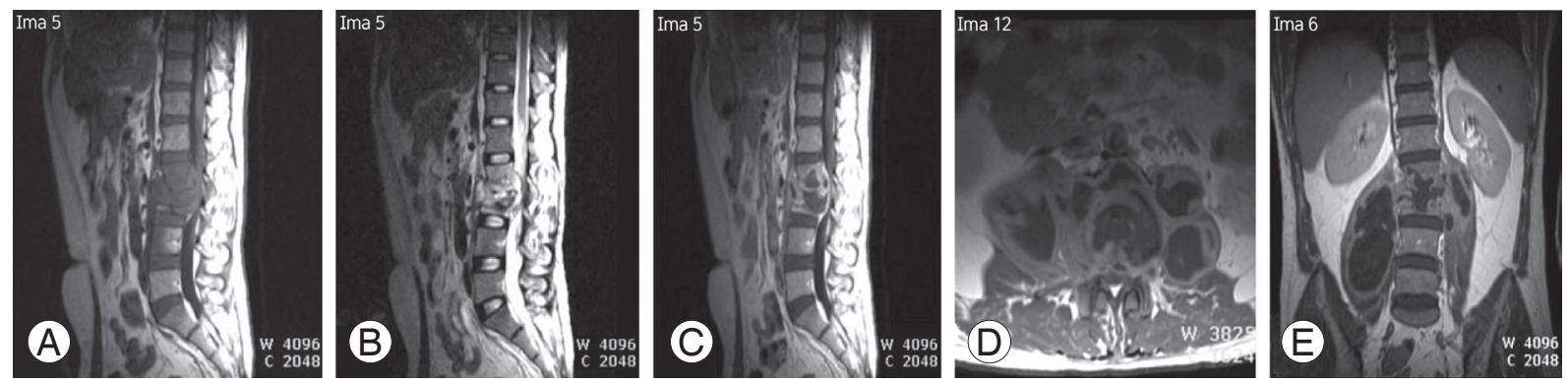

Fig. 1. Lumbar magnetic resonance imaging (MRI) of 35-year-old male with histological result was caseosa tuberculous spondylitis. (A) MRI results for T1WI sagittal image showed multiple hypointense lesions in the vertebral corpus and vertebral discs. (B, C) In the T2WI sagittal image, lesions appeared to be hyperintense accompanied by involvement of the intervertebral discs. (D) T1WI axial image with contrast showed intensified rim enhancement. (E) T1WI coronal image with contrast indicated a paravertebral abscess and bilateral psoas abscesses. T1WI, T1-weighted images; T2WI, T2-weighted images.
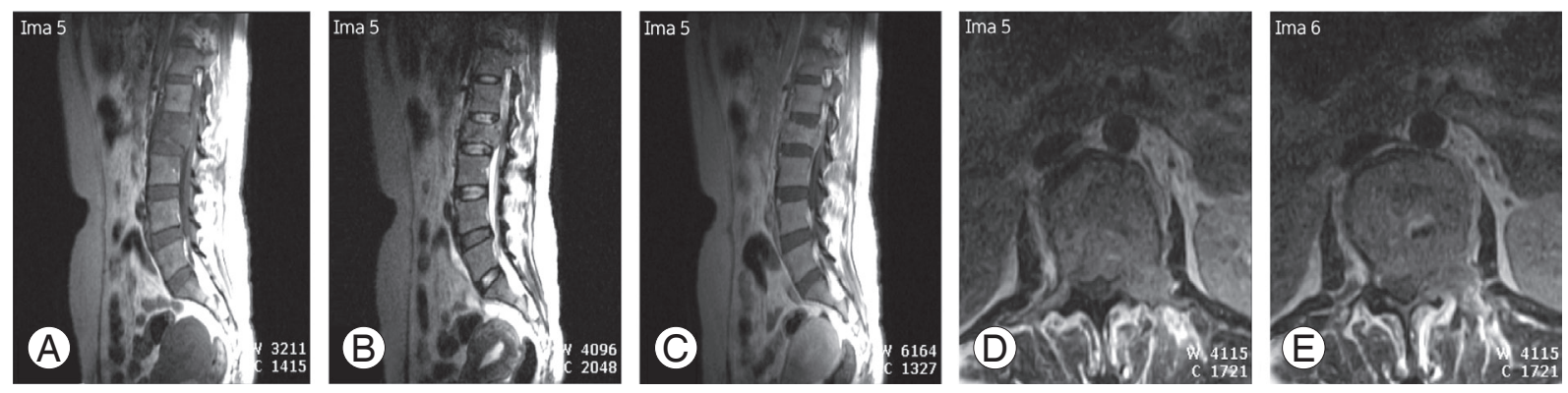

Fig. 2. Lumbar magnetic resonance imaging of 45-year-old female with histological result was metastatic adenocarcinoma. (A) T1Wl sagittal image showed hypointense lession (B) T2WI showed hyperintense lession, and (C) T1WI with contrast showed heterogeneously enhancement on L2 vertebral body, with central compression and narrowing of the spinal canal, did not seem to indicate involvement of the intervertebral discs. (D, E) The T1WI axial images with contrast indicated the involvement of paravertebral soft tissues and the left side of the pedicle which intensified heterogeneously with increased contrast. T1WI, T1-weighted images; T2WI, T2-weighted images.

distribution by age, spondylitis occurred more frequently in young adults with an average age of 38 years. Meanwhile, metastasis more frequently developed in older aged adults, i.e., with an average age of 52 years. Previous research results indicated that there were differences in the incidence by the age characteristics between tuberculous spondylitis in endemic countries, in which many events occur in children and young adults, and in non-endemic countries, in which tuberculous spondylitis is more common in adults $[8,9,16]$. Further, the research also showed 
that vertebral metastasis more commonly occurs in older aged adults in accordance with other studies which suggested that malignancy is more frequenty detected in older aged adults $[3,4,17,18]$.

With respect to the signal intensity of spondylitis on T1WI and T2WI images, the T1WI signal intensity was mostly hypointense, i.e., with a prevalence of $86.4 \%$, whereas the T2WI signal intensity was mostly hyperintense, with a prevalence of $90.9 \%$. In accordance with the pathophysiology of infections, early signs of spondylitis were inflammatory process, edema, and hyperemia. Initial response to infection which reached the vertebral corpus was the accumulation of fluid in the bone marrow. MRI images showed fluid accumulation or edema in the bone marrow long before the occurrence of bone destruction. The infection process started in the area around the vertebral endplate which manifested as a decrease in T1WI signal intensity and an increase in T2WI signal intensity in bone marrow and involved intervertebral discs $[1,6,8]$.

T1WI signal intensity of most vertebral metastases was hypointense, with a prevalence of $76.9 \%$. The prevalence of hyperintense and isointense T2WI signal intensity of vertebral metastasis was the same, namely $38.5 \%$. Pathophysiology of the metastatic process shows osteolysis, osteoblastic lesion, or mixed osteolytic and osteoblastic lesion. Differences in this type will provide an overview of different imaging in either the T1WI sequence or the T2WI sequence. In the osteolytic lesion type of metastasis, T1WI hypointensity and T2WI hyperintensity were observed. In the osteoblastic type of lesion, T1WI hypointensity and T2WI hypointensity were observed. Metastatic lesions were easily detectable on T1WI as a lowintensity signal, while on T2WI, hyperintense metastatic lesions were clearer than bone marrow due to the higher water content. At times, the presence of the halo sign and diffuse hyperintensity signal could be strong indicators of metastasis $[3,4,14,19]$.

Also, $81.8 \%$ of T1WI sequences with increased contrast to spondylitis showed significant intensification in the rim (rim enhancement). Contrast intensified pattern of this type is called paravertebral abcesses if it occurs in soft tissues. Initial phase of visible diffuse contrast intensification is called the cellulitis phase or granulomatous tissue which indicates the phlegmon stage. The second phase of contrast intensification pattern into the rim enhancement did not increase as it consisted of a set of abscess fluid surrounded by a thick wall of granulation tissues. Spon- dylodiscitis, epidural and paravertebral abscesses could be more easily detected on the T2WI sequence $[5,10]$.

The most common metastatic intensification pattern was heterogeneous, i.e., $80.0 \%$ and rim enhancement was almost not observed. Increasing the contrast on T1 fat saturation was rated as superior due to metastatic lesions which became more prominent and clearly visible after the suppression of the background surrounding fat $[6,9,12]$.

Short inversion time inversion recovery (STIR) sequence examination was not performed in all samples. Since data were retrieved retrospectively, action of manipulation of the STIR sequence could not be performed in all samples. Of the 35 samples, only 10 samples underwent STIR examination on sagittal cuts. All lesions exhibited hyperintense signals, both in spondylitis and metastasis. STIR is the most sensitive sequence for detecting the presence of abnormal lesions in bone marrow either in spondylitis or metastasis. This is because STIR can effectively suppress fat. As a result, elimination of the fat component in the vertebrae containing marrow fat in adults could be done. Spondylitis and metastatic lesions could appear more clearly as an area of hyperintensity in the background area of hypointensity since the fat component in the vertebral marrow fat had been eliminated [6].

The results of the research on the characteristics of involved vertebral corpus showed that spondylitis more commonly affected the anterior vertebral component (corpus), with a prevalence of $81.8 \%$. Spondylitis affected the anterior and posterior parts (pedicle, lamina, processus spinosus, and processus transversus) with a prevalence of $18.2 \%$. In contrast, the involvement of the anterior-posterior component in metastasis was greater, with a prevalence of $92.3 \%$ compared to the prevalence of $7.7 \%$ for the involvement of the anterior component alone. A preview of cases of spondylitis showed that the anterior corpus is often involved earlier, prior to posterior sides in which the intervertebral discs also involved. This can be explained by examining the spread of infection in the pathophysiology of spondylitis, which often occurs hematogeneously through the arterial system. Vertebral vascularization originated from intercostal arteries, vertebral arteries, lumbar arteries, and sacral arteries, which were located on the anterolateral surface of the vertebral corpus, and then they anastomosed with the arteries of the adjacent vertebrae. The spread of spondylitis infection occurred hematogeneously through the arterial line; 
hence, the first affected part was the anterior side of the vertebral corpus and endplate which has rich vascularity. Ongoing disease progression can cause side anterior vertebral collapse, leading to the formation of a wedge shape anteriorly, and the development of vertebral deformity angulation and gibbus. Subsequent deployment through discs allowed an overview of spondylodiscitis and then of the successive vertebral corpus $[10,20,21]$.

The results of this research indicated that there was no significant difference between radiological diagnosis and histological diagnosis in differentiating between spondylitis and metastasis. While assessing spondylitis, radiological diagnosis resulted in an error in three samples (13.6\%) as it was shown as metastasis when histopathologic diagnosis was spondylitis. All samples of metastasis (100\%) could be accurately assessed by radiological delineation when confirmed by histological results as metastasis. Thus, it can be stated that when the samples were radiologically diagnosed as metastasis, there was a greater possibility that the process was metastasis.

Errors were found in three samples of spondylitis, which were incorrectly assessed as metastasis on MRI examination due to the STIR sequence which was performed in those samples. As a result, lesion expansion and lesion characteristics were incomplete. As explained previously, the examination with STIR sequences is the most sensitive in assessing changes in bone marrow in the early phase. Due to the incompleteness of the STIR sequence, the diagnostic capabilities of MRI were reduced. Errors in assessment of spondylitis diagnosis occurred since there were some atypical findings in images of spondylitis in the samples, which were absence of disc involvement, skip lesions, and the presence of only one affected vertebral corpus. Images of spondylitis that did not involve the discs and there was presence of skip lesions were often mistakenly interpreted as images of metastasis. Absence of discitis was mainly identified in the vertebral infection caused by tuberculosis. The lack of proteolytic enzymes, especially in mycobacterium tuberculosis infection was the reason for non-involvement of discs. Erosion on the anterior surface of the vertebral corpus without disc involvement or discitis was considered to be an atypical case. The spread of infection through the subligament caused further vertebral involvement, not only in the anterolateral side of the vertebral corpus, but also in the posterior side of the vertebral corpus through the posterior longitudinal ligament. The subligamentous spread of infection could explain the occurrence of skip lesions in spondylitis $[8,13,14]$.

Images of spondylitis with central type of compression of the vertebral corpus without the involvement of discs formed a picture of vertebra plana with the involvement of non-contiguous vertebral components (skip lesions) which made it difficult to differentiate it from metastasis. Central compression type in spondylitis was explainable from the pathophysiology viewpoint due to the spread of infection through Batson's venous plexus which was different from the spread of infectious spondylitis in general, i.e., through the arterial system. An atypical image often causes difficulties in determining the imaging diagnosis. In areas of endemic tuberculosis, suspicion of spondylitis should be an important consideration and it should be proven by histological biopsy for establishing the diagnosis [5,14-16].

This study has several limitations. The first limitation is rectrospective samples, no manipulative actions for retrieving the sequence or cuts could be taken. Thus, STIR sequence retrieval action could not be performed in some samples. Another limitation of the study is since most MRI images were diagnosed as both spondylitis and metastasis, not all of them were examined histologically, although histology examination alone is considered the gold standard for the diagnosis. Histological results of some samples were assessed as dubious and atypical for the images of both spondylitis and metastasis; therefore, they had to be excluded from the analysis.

\section{Conclusions}

MRI has a high suitability with histological results in differentiating spondylitis from vertebral metastasis with a tendency that MRI is more accurate in diagnosing metastasis.

\section{Conflict of Interest}

No potential conflict of interest relevant to this article was reported.

\section{References}

1. Pierre-Jerome C, Arslan A, Bekkelund SI. MRI of the spine and spinal cord: imaging techniques, normal anatomy, artifacts, and pitfalls. J Manipulative Physiol Ther 2000;23:470-5. 
2. WebMD. Magnetic resonance imaging [Internet]. New York: WebMD; c2015 [cited 2015 May 20]. Available from: http://www.webmd.com/a-to-zguides/magnetic-resonance-imaging-mri.

3. Ecker RD, Endo T, Wetjen NM, Krauss WE. Diagnosis and treatment of vertebral column metastases. Mayo Clin Proc 2005;80:1177-86.

4. Andreula C, Murrone M. Metastatic disease of the spine. Eur Radiol 2005;15:627-32.

5. Sobottke R, Seifert H, Fätkenheuer G, Schmidt M, Gossmann A, Eysel P. Current diagnosis and treatment of spondylodiscitis. Dtsch Arztebl Int 2008;105:181-7.

6. Khalid M, Siddiqui MA, Qaseem SM, Mittal S, Iraqi AA, Rizvi SA. Role of magnetic resonance imaging in evaluation of tubercular spondylitis: pattern of disease in 100 patients with review of literature. JNMA J Nepal Med Assoc 2011;51:116-21.

7. Khaw FM, Worthy SA, Gibson MJ, Gholkar A. The appearance on MRI of vertebrae in acute compression of the spinal cord due to metastases. J Bone Joint Surg Br 1999;81:830-4.

8. Jung NY, Jee WH, Ha KY, Park CK, Byun JY. Discrimination of tuberculous spondylitis from pyogenic spondylitis on MRI. AJR Am J Roentgenol 2004;182: 1405-10.

9. Chang MC, Wu HT, Lee CH, Liu CL, Chen TH. Tuberculous spondylitis and pyogenic spondylitis: comparative magnetic resonance imaging features. Spine (Phila Pa 1976) 2006;31:782-8.

10. Dziurzyńska-Białek E, Kruk-Bachonko J, Guz W, Losicki M, Krupski W. Diagnostic difficulties resulting from morphological image variation in spondylodiscitis MR imaging. Pol J Radiol 2012;77:25-34.
11. Kim JK, Learch TJ, Colletti PM, Lee JW, Tran SD, Terk MR. Diagnosis of vertebral metastasis, epidural metastasis, and malignant spinal cord compression: are T1-weighted sagittal images sufficient? Magn Reson Imaging 2000;18:819-24.

12. Shah LM, Salzman KL. Imaging of spinal metastatic disease. Int J Surg Oncol 2011;2011:769753.

13. Kleinstuck F, Dvorak J, Mannion AF. Are "structural abnormalities" on magnetic resonance imaging a contraindication to the successful conservative treatment of chronic nonspecific low back pain? Spine (Phila Pa 1976) 2006;31:2250-7.

14. Zimmerli W. Clinical practice: vertebral osteomyelitis. N Engl J Med 2010;362:1022-9.

15. Jaramillo-de la Torre JJ, Bohinski RJ, Kuntz C 4th. Vertebral osteomyelitis. Neurosurg Clin N Am 2006; 17:339-51.

16. Garg RK, Somvanshi DS. Spinal tuberculosis: a review. J Spinal Cord Med 2011;34:440-54.

17. Rose PS, Buchowski JM. Metastatic disease in the thoracic and lumbar spine: evaluation and management. J Am Acad Orthop Surg 2011;19:37-48.

18. Maccauro G, Spinelli MS, Mauro S, Perisano C, Graci C, Rosa MA. Physiopathology of spine metastasis. Int J Surg Oncol 2011;2011:107969.

19. Aebi M. Spinal metastasis in the elderly. Eur Spine J 2003;12 Suppl 2:S202-13.

20. Harada Y, Tokuda O, Matsunaga N. Magnetic resonance imaging characteristics of tuberculous spondylitis vs. pyogenic spondylitis. Clin Imaging 2008;32:303-9.

21. Lee KY. Comparison of pyogenic spondylitis and tuberculous spondylitis. Asian Spine J 2014;8:216-23. 